\title{
Primary pelvic exenteration: Our experience with 23 patients from a single institution
}

\author{
MIHAI GHEORGHE ${ }^{1}$, ALEXANDRA LAVINIA COZLEA ${ }^{1}$, SZILARD LEO KISS ${ }^{1}$, MIHAI STANCA ${ }^{1}$, \\ MIHAI EMIL CĂPÎLNA ${ }^{1}$, NICOLAE BACALBAȘA ${ }^{2}$ and ANDREEA ANAMARIA MOLDOVAN ${ }^{3}$
}

\begin{abstract}
${ }^{1}$ First Obstetrics and Gynecology Clinic, 'George Emil Palade' University of Medicine, Pharmacy, Science and Technology, 540136 Târgu Mureș; ${ }^{2}$ Department of Obstetrics and Gynecology, 'Carol Davila' University of Medicine and Pharmacy, 020022 Bucharest; ${ }^{3}$ Department of Infectious Diseases, Brașov County Emergency Hospital, 500326 Brașov, Romania
\end{abstract}

Received May 24, 2021; Accepted June 23, 2021

DOI: $10.3892 / \mathrm{etm} .2021 .10494$

\begin{abstract}
This study was designed with an aim to share our experience of primary pelvic exenterations. The study included 23 patients with different types of pelvic cancer enrolled at a single institution between November 2011 and July 2020. The patient mean age was 55 years (range, $43-72$ years) and the oncological indications included: Stage IVa cervical cancer (11 cases, $48.9 \%$ ), stage IVa endometrial cancer (1 case, $4.3 \%$ ), stage IVa vaginal cancer (6 cases, 26\%), stage IIIb bladder cancer ( 3 cases, $13 \%$ ), stage IIIc rectal cancer (1 case, $4.3 \%$ ) and undifferentiated pelvic sarcoma ( 1 case, $4.3 \%)$. Total, anterior, and posterior pelvic exenterations were performed on $34.4,56.5$ and $13 \%$ of cases, respectively. Related to levator ani muscle, $13(56.5 \%)$ pelvic exenterations were supralevatorian, 10 (43.5\%) infralevatorian, and 5 (21.7\%) were infralevatorian with vulvectomy. No major intraoperative complications occurred. Seven patients (30.5\%) developed early complications, 4 of them (17.4\%) required reoperation and $1(4.3 \%)$ perioperative death caused by a pulmonary embolism was recorded. Only 1 patient experienced a late complication, a urostomy stenosis. Over a median follow-up period of 35 months, 8 (34.8\%) patients died. The median overall survival (OS) was 33 months (range, 1-96 months). The 2-year and 5-year survival rates were 72 and $66 \%$, respectively. Primary pelvic exenteration may be related with various postoperative complications, without high perioperative morality and with long-term survival.
\end{abstract}

Correspondence to: Dr Mihai Gheorghe, First Obstetrics and Gynecology Clinic, 'George Emil Palade' University of Medicine, Pharmacy, Science and Technology, 50 Gheorghe Marinescu Street, 540136 Târgu Mureș, Romania

E-mail: mihai18go@gmail.com

Key words: pelvic exenteration, pelvic malignancies, primary, complications, survival

\section{Introduction}

In patients with advanced primary or recurrent gynecologic (1), urologic or rectal cancers without metastatic disease, extensive aggressive surgery such as pelvic exenteration may be necessary for curative intent treatment (2).

Brunschwig was the first to describe pelvic exenteration in the 1940s (3). Exenteration was initially considered as a palliative procedure for patients with extensive pelvic cancers, with an extremely high perioperative mortality rate of $23 \%$. After significant progress related to patient selection, improvements in operative techniques and intensive care, the mortality rate has decreased to $3-9 \%$ as documented in more recent studies $(4,5)$, and a 5-year survival rate between 20 and $60 \%$ (6-14) with a good quality of life (15).

Primary pelvic exenteration is considered as a first-line radical surgical procedure for patients with advanced pelvic malignancies, prior to any oncological treatment. It may be performed in patients with stage IVa gynecological cancers (1), tumor-associated urogenital or rectogenital fistulas, and in some cases, when the histology of the disease (soft tissue sarcomas, neuroendocrine tumors) predicts chemoradiation therapy resistance (13). This procedure may also be performed in rare malignant conditions such as synchronous pelvic cancers (16).

The procedure may be classified as total (removal of the tumor together with the uterus, vagina, urinary bladder and rectum), anterior (bladder, uterus and vagina) and posterior (rectum, uterus and vagina). In relation to the levator ani muscle, the procedure is classified as supralevatorian, infralevatorian or infralevatorian with vulvectomy. The surgical procedure includes an exenterative phase followed by a reconstructive phase consisting of a continent or incontinent urinary diversion, definitive end colostomy or low rectal anastomosis or and vaginal and pelvic floor reconstruction $(17,18)$.

The aim of the present study was to analyze our primary pelvic exenteration experience from a single institution in patients with locally advanced primary pelvic malignancies.

\section{Materials and methods}

This study is a retrospective analysis of all patients who underwent primary pelvic exenteration for advanced pelvic cancer 
in a tertiary university hospital in Târgu Mures, Romania. The study was approved by the Ethics Medical Committee of the University Hospital of Târgu Mures, (protocol code 27227, 10/03/2020). Twenty-three primary exenterations were performed between August 2011 and July 2020. Informed consent was obtained from every case, and all patients were evaluated by the anesthesiology team in order to evaluate their medical condition to support a complex surgical intervention. All procedures were performed with a curative intent, not for palliation purposes. None of the patients submitted to primary pelvic exenteration received neoadjuvant treatment. The exenteration was the first therapeutic approach. For exclusion of all oncological contraindications for pelvic exenteration and assessment of operability, the preoperative work-up included a mandatory transvaginal or transrectal echography plus magnetic resonance imaging (MRI) or computed tomography (CT). All patients proposed for a total or anterior exenteration underwent cystoscopy, or a colonoscopy for total or posterior exenteration. Among the 27 patients identified, pelvic exenteration was abandoned in 4 patients due to oncological contraindications encountered during surgery: Pelvic sidewall involvement of the tumor with extension to the bony structures and involvement of the neurovascular structures of the sciatic foramen in 1 patient, or the detection of peritoneal carcinomatosis in 3 cases, which had not been described in the preoperative imaging work-up. Data were collected from the medical records and consisted of patient demographics, types of malignancy, details on intraoperative management provided, postoperative complications and follow-up. The presence of postoperative complications were assessed according to the Clavien-Dindo scale (19).

Statistical analysis. Statistical analysis was performed using the SPSS 21.0 statistical package (IBM Corp.). Quantitative variables are presented as mean and median, while qualitative and categorical variables are expressed both as integer and percentage values. Survival curve was calculated using the Kaplan-Meier method.

\section{Results}

Epidemiological and preoperative clinical characteristics. Over a period of 9 years, 23 patients underwent primary exenteration for locally advanced stage pelvic cancers. The median patient age at the time of surgery was 55 years (range, 43-72 years). The origin of the primary tumor included stage IVa cervical cancer (11 cases, 48.9\%), stage IVa endometrial cancer (1 case, $4.3 \%$ ), stage IVa vaginal cancer (6 cases, $26 \%$ ), stage IIIb bladder cancer (3 cases, 13\%), stage IIIc rectal cancer (1 case, $4.3 \%)$ and undifferentiated pelvic sarcoma (1 case, $4.3 \%$ ) (Table I). Ten out of the 17 patients with stage IVa cervical or vaginal cancer had already developed a vesico-vaginal ( 8 women) or recto-vaginal ( 2 women) fistula at the moment of surgery; also, the patient with stage IIIc rectal cancer had developed a recto-vaginal fistula.

Procedures and complications of pelvic exenteration. As the type of exenterative procedure was related to the tumor involvement of pelvic organs, 7 (30.5\%) patients required total exenteration, $13(56.5 \%)$ procedures were anterior and
$3(13 \%)$ were posterior exenterations. Regarding the levator ani muscle, with the aim to obtain tumor-free resection margins, $13(56.5 \%)$ pelvic exenterations were supralevatorian, 10 $(43.5 \%)$ infralevatorian, and $5(21.7 \%)$ were infralevatorian with vulvectomy.

Among the 10 patients with total or posterior pelvic exenterations, a low rectal anastomosis was performed in 3 cases and in 7 patients an end definitive colostomy was conducted due to insufficient unaffected rectal stump. Urinary diversion procedures were performed for all patients who underwent a total or anterior exenteration, tailoring a Bricker's ileal (in 15 patients) or sigmoid (in 5) incontinent conduit (20), technically easier to perform compared to other urinary diversion procedures and also associated with lower rates of postoperative complications. The option for an ileal or sigmoid urinary conduit after total exenteration is dependent on the remaining length of the sigmoid colon and on the avoidance of an unnecessary ileal anastomosis needed for the ileal conduit. In all anterior exenterations, an ileal conduit was performed. All ureteric-enteral anastomoses were adjusted on 'double J' ureteral stents in order to prevent a subsequent stenosis. The median length of surgery (364 min), median estimated blood loss $(610 \mathrm{ml})$ and the need for transfusion in our series are documented in Table I.

All of the patients were maintained in the intensive care unit for more than 4 days for close monitoring due to the complexity of the procedure and for postoperative therapy as antithrombotic prophylaxis, total parenteral nutrition, prophylactic antibiotic treatment and intravenous albumin administration.

Upon final pathology report, clear resection margins were achieved only in 19 out of 23 patients $(86.2 \%)$. All 5 patients with positive margins were sent for adjuvant chemotherapy.

No major intraoperative complications occurred. Postoperative complications were characterized using the Clavien-Dindo classification (19). Seven patients $(30.5 \%)$ experienced early complications and 1 patient presented with late complication, respectively (Table II). Among the early complications, one Clavien-Dindo grade $\mathrm{V}$ was registered, a patient 46 years of age, referred to the hospital for stage IIIB bladder cancer. This patient underwent an anterior supralevatorian exenteration, with no intraoperative complications, but experienced sudden death on the 16th postoperative day due to a pulmonary embolism after home discharge. Four patients experienced Clavien-Dindo grade IIIb complications: Enteric fistulas-3 ileal fistulas with peritonitis and one entero-vaginal fistula, all necessitating re-laparotomies and ileum re-anastomosis. Two patients who underwent infralevatorian exenteration with vulvectomy developed a perineal wound infection with tissue necrosis, necessitating prolonged local treatment (Clavien-Dindo grade II). Only one patient has experienced a late complication: A ureteric-enteral stenosis solved finally by a unilateral permanent percutaneous nephrostomy.

Survival outcomes. Over a median follow-up period of 35 months, 8 (34.8\%) patients died. The median overall survival (OS) was 33 months (range, 1-96 months) (Fig. 1). The 2-year and 5-year survival rates were 72 and $66 \%$, respectively. 
Table I. Demographic characteristics and intraoperative details of the patients undergoing exenterations.

Characteristics/intraoperative details

Mean age (range) in years

Origin of malignancy, n $(\%)$

Cervical

Endometrial

Vaginal

Bladder

Rectum

Undifferentiated pelvic sarcoma, n (\%)

Type of exenteration regarding topography, n (\%)

Total

Anterior

Posterior

Type of exenteration regarding the levator ani muscle, n (\%)

Supralevatorian

Infralevatorian

Infralevatorian with vulvectomy

Type of urinary tract reconstruction, $\mathrm{n}(\%)$

Non-continent urinary conduit type Bricker

Type of bowel reconstruction, n (\%)

Colostomy

Colorectal anastomosis

Length of surgery (min), median (range)

Estimated blood loss (ml), median (range)

Transfusion volumes (ml), median (range)

Hospital stay after PE (days), median (range)
Data values

$53.5(43-72)$

Stage/histological type

Iva/squamous cell carcinoma

$11(48.9 \%)$

Iva/adenocarcinoma

$1(4.3 \%)$

Iva/squamous cell carcinoma

$6(26 \%)$

IIIb/urothelial carcinoma

$3(13 \%)$

IIIc/adenocarcinoma

$1(4.3 \%)$

$1(4.3 \%)$

$7(30.5 \%)$

$13(56.5 \%)$

$3(13 \%)$

$13(56.5 \%)$

$10(43.5 \%)$

$5(21.7 \%)$

$20(87 \%)$

$7(30.5 \%)$

$3(13 \%)$

364 (270-560)

$600(300-2,100)$

$700(0-1,800)$

$20(11-75)$

Table II. Early and late complications and survival outcomes of the patients $(n=23)$ after pelvic exenteration.

Complications

Early complications

Clavien-Dindo grade II

Clavien-Dindograde IIIb

Clavien-Dindograde V

Late complications

Urostomy stenosis

Survival outcomes

Alive, free of disease

Deceased
Total study group data [n (\%)]

4 (17.4)

$1(4.3)$

15 (65.2)

8 (34.8)

\section{Discussion}

Although pelvic exenteration is originally intended as a palliative procedure, currently it is performed with curative intent for the treatment of pelvic disease (rectal, cervical, endometrial, vaginal, bladder and soft-tissue sarcoma) (13). The most important parameter in the evaluation of risk related to the operative procedure is the mortality rate. Since the initial description, mortality rates have improved from higher than $30 \%$ to more acceptable rates of 0 to $10 \%$ with 5-year OS varying between 20 and 60\%, despite the high morbidity rate $(5-14,21-24)$. In the present study, there was one perioperative death $(4.3 \%)$ due to a sudden pulmonary embolism, despite prophylactic anticoagulant protocol during hospitalization and home discharge and patient early mobilization. 


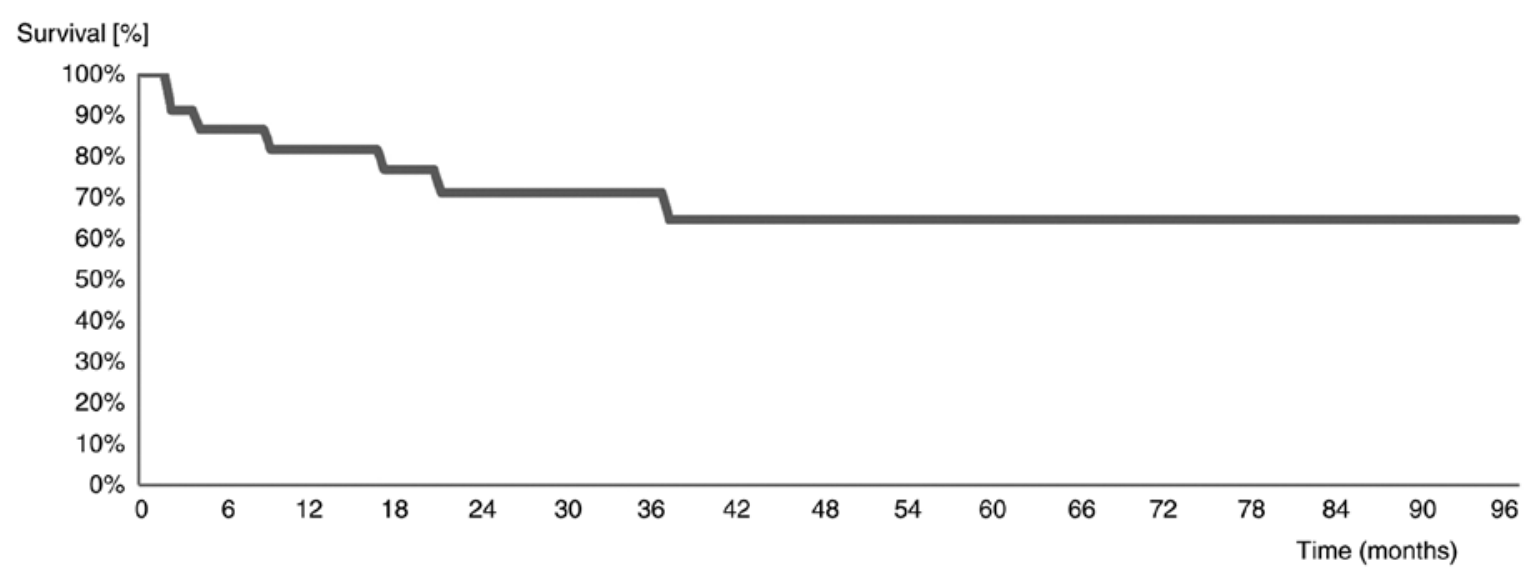

Figure 1. Kaplan-Meier curve showing overall survival of the study group $(\mathrm{n}=23)$.

The role of pelvic exenteration for pelvic recurrences after gynecologic, urinary or rectal cancers for patients previously irradiated, when no other therapeutic options with curative intent are available, is well established. In contrast, there is a continued debate between oncologic surgeons and medical oncologists or radiotherapists regarding primary pelvic exenterations. The evidence is scarce and only a few studies on this topic have been published $(1,2,7-11,13,14)$. In our study, 11 out of the 23 patients (47.8\%) with primary pelvic exenterations had fistulas at the moment of surgery, a condition that inevitably alters the quality of life of these patients and that will not be solved by oncologic treatment. The 5 -year OS after pelvic exenterations which ranges between 20 and $60 \%$ in all studies, when the oncological indications and contraindications are respected (5-14,22-26), is similar or higher compared to the OS after chemoradiotherapy for these advanced pelvic cancers, considered separately. Kramer et al (25) reported that $22 \%$ of his patients who underwent radiochemotherapy for locally advanced cervical cancer with curative intent developed fistulas, and the 5-year survival rate was $18 \%$. Moore et al (26) reported that fistulas appeared as a complication of radiochemotherapy in $48 \%$ of his patients.

For all our primary pelvic exenteration patients, a 5-year survival probability of $43 \%$ was calculated, which is similar to the rates found in other studies on exenteration $(6-14,27)$. Also, the morbidity after exenteration was comparable: In our study, 4 patients (17\%) had to endure a second surgery due to bowel fistulas. Other early minor complications in our series included perineal wound infection in $2(8.7 \%)$ patients. Late postoperative complications were noted in one case (4.3\%), presenting an urostomy stenosis. The relatively high 5-year OS and low morbidity after the procedure are strong arguments in favor of primary pelvic exenteration. The current series supports the increasing number of studies regarding the role of pelvic exenteration for selected patients with locally advanced primary pelvic malignancies (28).

In light of the associated morbidities, the aim of any exenterative surgery must include the achievement of tumor-free margins. Existing tumors that are fixed to the lower pelvic side wall have long been regarded as a contraindication for pelvic exenterations, but the role of laterally extended pelvic resection (LEER) in the surgical treatment of pelvic malignancies has been confirmed by some reports $(29,30)$. The only contraindication for LEER is the involvement of the sciatic nerve (31), but these pelvic side wall resections are technically difficult and may be associated with increased risks because of frequent anatomic anomalies (31).

Completeness of the tumor resection was the only variable with a significant impact on survival according to Zoucas et al (32). In our series, clear resection margins were achieved in only $82.6 \%$ of the patients. The inferior resection line (urethra, vagina and rectum) has been proven to be the weak point for the majority of patients with a positive microscopic resection line and this has to be pushed downwards as much as necessary to obtain clear margins.

An important element of every pelvic exenteration procedure, affecting the duration of surgery, the frequency and type of complication, and the postoperative quality of life, is the method used for urinary and/or fecal diversion. The Bricker procedure remains the most performed technique for urinary diversion (20). In our study group, this method was applied in all patients with anterior or total exenteration. But, in recent years, the low rectal anastomosis and orthotopic continent urinary diversions are more commonly used after pelvic exenterations, mainly for patients more fit for a prolonged surgery and without tumor involvement of the bladder neck or lower rectum. These surgical techniques avoid the need of external stomas and, subsequently, the patient quality of life is significantly improved (33).

The learning curve for pelvic exenterations is long for the entire involved team (gynecologic oncologist surgeon, anesthesiologist, radiologist, urologist). Comparing the early period when pelvic exenteration was implemented in our department, in recent years, the tendency is to lower significantly the operative time, the blood transfusion volume and the complication rate. After more than 80 pelvic exenterations (not only primary) already performed by our team, the shift towards implementation of Enhanced Recovery After Surgery (ERAS) protocols before, during and after pelvic exenteration has contributed to better outcomes for these extremely fragile patients with advanced pelvic malignancies, but this issue will be the subject of another future paper.

Major biases of our study are the retrospective nature of the analysis, the heterogeneity of the advanced pelvic cancers and the relatively small number of patients. Yet, these factors are present in the majority of series reported in the literature 
in regards to pelvic exenteration. Despite these limitations, our study has contributed to the evidence that primary pelvic exenteration is a feasible surgical option for selected patients with locally advanced pelvic malignancies accompanied by acceptable long-term outcomes. It is imperative to adopt a multidisciplinary approach when performing such technically demanding operations to achieve better outcomes for the patients. In the near future, considering the new data regarding the safety of minimally invasive surgery in the treatment of cervical cancer (34), the exenterative and partially the reconstructive phase might be performed by laparoscopic or robotic surgery.

Primary pelvic exenteration for locally advanced pelvic malignancies is accompanied by considerable morbidity, but with acceptable OS. The eligibility of patients for this radical surgical approach should be assessed by careful patients' selection, preoperative counseling and should be carried out only in surgical centers with well trained staff.

\section{Acknowledgements}

Not applicable.

\section{Funding}

No funding was received.

\section{Availability of data and materials}

The data that support the findings of this study are available from the corresponding author (MG), upon reasonable request.

\section{Authors' contributions}

MG contributed to the conception and design of the study, analysis and interpretation of the patient data. MEC contributed to the conception and design of the study and was the leading surgeon for all the surgical procedures described in our report. ALC, SLK and MS contributed to the acquisition of the patient data, the analysis and interpretation of the data of the study and the writing of the article. AAM and NB supervised the work and revised the article, contributed to the drafting of the work and its critical revision for important intellectual content. All authors read and approved the final manuscript and agree to be accountable for all aspects of the research in ensuring that the accuracy or integrity of any part of the work are appropriately investigated and resolved.

\section{Ethics approval and consent to participate}

The study was conducted according to the guidelines of the Declaration of Helsinki, and approved by the Ethics Medical Committee of the University Hospital of Târgu Mures, (protocol code 27227, 10/03/2020).

\section{Patient consent for publication}

This manuscript does not contain case details, personal information or images that may enable an individual to be identified.

\section{Competing interests}

The authors declare that they have no competing interests.

\section{References}

1. Ungar L, Palfalvi L and Novak Z: Primary pelvic exenteration in cervical cancer patients. Gynecol Oncol 111 (Suppl 2): S9-S12, 2008.

2. Maggioni A, Roviglione G, Landoni F, Zanagnolo V, Peiretti M, Colombo N, Bocciolone L, Biffi R, Minig L and Morrow CP: Pelvic exenteration: Ten-year experience at the European institute of oncology in Milan. Gynecol Oncol 114: 64-68, 2009.

3. Brunschwig A: Complete excision of pelvic viscera for abdominal carcinoma; a one-stage abdominoperineal operation with end colostomy and bilateral ureteral implantation into the colon above the colostomy. Cancer 1: 177-183, 1948.

4. Berek JS, Howe C, Lagasse LD and Hacker NF: Pelvic exenteration for recurrent gynecologic malignancy: Survival and morbidity analysis of the 45-year experience at UCLA. Gynecol Oncol 99: 153-159, 2005

5. Forner DM and Lampe B: Intestinal complications after pelvic exenterations in gynecologic oncology. Int J Gynecol Cancer 19: 958-962, 2009.

6. Khoury-Collado F, Einstein MH, Bochner BH, Alektiar KM, Sonoda Y, Abu-Rustum NR, Brown CL, Gardner GJ, Barakat RR and Chi DS: Pelvic exenteration with curative intent for recurrent uterine malignancies. Gynecol Oncol 124: 42-47, 2012.

7. Fotopoulou C, Neumann U, Kraetschell R, Schefold JC, Weidemann H, Lichtenegger W and Sehouli J: Long-term clinical outcome of pelvic exenteration in patients with advanced gynecological malignancies. J Surg Oncol 101: 507-512, 2010.

8. Schmidt AM, Imesch P, Fink D and Egger H: Indications and long-term clinical outcomes in 282 patients with pelvic exenteration for advanced or recurrent cervical cancer. Gynecol Oncol 125: 604-609, 2012.

9. Soper JT, Berchuck A, Creasman WT and Clarke-Pearson DL: Pelvic exenteration: Factors associated with major surgical morbidity. Gynecol Oncol 35: 93-98, 1989.

10. Anthopoulos AP, Manetta A, Larson JE, Podczaski ES, Bartholomew MJ and Mortel R: Pelvic exenteration: A morbidity and mortality analysis of a seven-year experience. Gynecol Oncol 35: 219-223, 1989.

11. Eisenkop SM and Spirtos NM: Procedures required to accomplish complete cytoreduction of ovarian cancer: Is there a correlation with 'biological aggressiveness' and survival? Gynecol Oncol 82: 435-441, 2001.

12. Marnitz S, Köhler C, Müller M, Behrens K, Hasenbein K and Schneider A: Indications for primary and secondary exenterations in patients with cervical cancer. Gynecol Oncol 103: 1023-1030, 2006.

13. Ferenschild FT, Vermaas M, Verhoef C, Ansink AC, Kirkels WJ, Eggermont AM and de Wilt JH: Total pelvic exenteration for primary and recurrent malignancies. World J Surg 33: 1502-1508, 2009.

14. Hawighorst-Knapstein S, Fusshoeller C, Franz C, Trautmann K, Schmidt M, Pilch H, Schoenefuss G, Knapstein PG, Koelbl H, Kelleher DK and Vaupel P: The impact of treatment for genital cancer on quality of life and body image-results of a prospective longitudinal 10-year study. Gynecol Oncol 94: 398-403, 2004.

15. Höckel Mand Dornhöfer N: Pelvic exenteration for gynaecological tumours: Achievements and unanswered questions. Lancet Oncol 7: 837-847, 2006.

16. Căpîlna ME, Rusu SC, Laczko C, Szabo B and Marian C: Three synchronous primary pelvic cancers-a case report. Eur J Gynaecol Oncol 36: 216-218, 2015.

17. PelvExCollaborative: Pelvic exenteration for advanced nonrectal pelvic malignancy. Ann Surg 270: 899-905, 2019.

18. Chiva LM, Lapuente F, González-Cortijo L, González-Martín A, Rojo A, García JF and Carballo N: Surgical treatment of recurrent cervical cancer: State of the art and new achievements. Gynecol Oncol 110 (3 Suppl 2): S60-S66, 2008.

19. Dindo D, Demartines N and Clavien PA: Classification of surgical complications: A new proposal with evaluation in a cohort of 6336 patients and results of a survey. Ann Surg 240: 205-213, 2004.

20. Bricker EM: Bladder substitution after pelvic evisceration. Surg Clin North Am 30: 1511-1521, 1950. 
21. Căpîlna ME, Moldovan B and Szabo B: Pelvic exenteration-our initial experience in 15 cases. Eur J Gynaecol Oncol 36: 142-145, 2015.

22. Bacalbasa N, Balescu I, Vilcu M, Neacsu A, Dima S, Croitoru A and Brezean I: Pelvic exenteration for locally advanced and relapsed pelvic malignancies-an analysis of 100 cases. In Vivo 33: 2205-2210, 2019

23. Lewandowska A, Szubert S, Koper K, Koper A, Cwynar G and Wicherek L: Analysis of long-term outcomes in 44 patients following pelvic exenteration due to cervical cancer. World J Surg Onc 18: 234, 2020.

24. Goldberg JM, Piver MS, Hempling RE, Aiduk C, Blumenson L and Recio FO: Improvements in pelvic exenteration: Factors responsible for reducing morbidity and mortality. Ann Surg Oncol 5: 399-406, 1998.

25. Kramer C, Peschel RE, Goldberg N, Kohorn EI, Chambers JT, Chambers SK and Schwartzet PE: Radiation treatment of FIGO stage IVA carcinoma of the cervix. Gynecol Oncol 32: 323-326, 1989.

26. Moore KN, GoldMA, McMeekin DS andZorn KK: Vesicovaginal fistula formation in patients with stage IVA cervical carcinoma. Gynecol Oncol 106: 498-501, 2007.

27. Forner DM and Lampe B: Exenteration as a primary treatment for locally advanced cervical cancer: Long-term results and prognostic factors. Am J Obstet Gynecol 205: 148.e1-e6, 2011.

28. Ahmadi N, Tan KK, Solomon MJ, Al-Mozany N and Carter J: Pelvic exenteration for primary and recurrent gynecologic malignancies is safe and achieves acceptable long-term outcomes. J Gynecol Surg 30: 255-259, 2014.

29. Höckel M, Horn LC and Einenkel J: (Laterally) extended endopelvic resection: Surgical treatment of locally advanced and recurrent cancer of the uterine cervix and vagina based on ontogenetic anatomy. Gynecol Oncol 127: 297-302, 2012
30. Li L, Ma SQ, Tan XJ, Zhong S and Wu M: Pelvic exenteration for recurrent and persistent cervical cancer. Chin Med J (Engl) 131: 1541-1548, 2018.

31. Căpîlna ME, Szabo B, Rusu SC, Becsi J, Moldovan B, Neagoe RM and Muhlfay G: Anatomical variations of the obturator veins and their surgical implications. Eur J Gynaecol Oncol 38: 263-265, 2017.

32. Zoucas E, Frederiksen S, Lydrup ML, Månsson W, Gustafson P and Alberius P: Pelvic exenteration for advanced and recurrent malignancy. World J Surg 34: 2177-2184, 2010.

33. Andikyan V, Khoury-Collado F, Gerst SR, Talukdar S, Bochner BH, Sandhu JS, Abu-Rustum N, Sonoda Y, Barakat RR and Chi DS: Anterior pelvic exenteration with total vaginectomy for recurrent or persistent genitourinary malignancies: Review of surgical technique, complications, and outcome. Gynecol Oncol 126: 346-350, 2012.

34. Chiva L, Zanagnolo V, Querleu D, Martin-Calvo N, Arévalo-Serrano J, Căpîlna ME, Fagotti A, Kucukmetin A, Mom C, Chakalova G, et al: SUCCOR study: An international European cohort observational study comparing minimally invasive surgery versus open abdominal radical hysterectomy in patients with stage IB1 cervical cancer. Int J Gynecol Cancer 30: $1269-1277,2020$

This work is licensed under a Creative Commons Attribution-NonCommercial-NoDerivatives 4.0 International (CC BY-NC-ND 4.0) License. 